\title{
Large enhancement of the third-order optical susceptibility in Cu-silica composites produced by low-energy high-current ion implantation
}

\author{
J. Olivares, ${ }^{\text {a) }}$ J. Requejo-Isidro, R. del Coso, R. de Nalda, J. Solis, and C. N. Afonso
}

Instituto de Optica, CSIC, Serrano 121, 28006 Madrid, Spain

\author{
A. L. Stepanov, ${ }^{\text {b) }}$ D. Hole, and P. D. Townsend \\ EIT, University of Sussex, Brighton, BN1 9QH, United Kingdom
}

\author{
A. Naudon \\ Laboratoire de Métallurgie Physique, UMR 6630 CNRS, Université de Poitiers, SP2MI, Bd. M. et P. Curie, \\ BP 30179 Futuroscope Cedex, France
}

(Received 11 December 2000; accepted for publication 22 April 2001)

\begin{abstract}
Low-energy high-current ion implantation in silica at a well-controlled substrate temperature has been used to produce composites containing a large concentration of spherical $\mathrm{Cu}$ clusters with an average diameter of $4 \mathrm{~nm}$ and a very narrow size distribution. A very large value for the third-order optical susceptibility, $\chi^{(3)}=10^{-7}$ esu, has been measured in the vicinity of the surface plasmon resonance by degenerate four-wave mixing at $585 \mathrm{~nm}$. This value is among the largest values ever reported for $\mathrm{Cu}$ nanocomposites. Additionally, the response time of the nonlinearity has been found to be shorter than 2 ps. The superior nonlinear optical response of these implants is discussed in terms of the implantation conditions. (c) 2001 American Institute of Physics.
\end{abstract}

[DOI: $10.1063 / 1.1379772]$

In recent years there has been a growing interest in composite glasses containing metal nanoclusters for their potential application in all-optical switching devices. These composites show a large third-order optical nonlinear response associated to various phenomena, i.e. dielectric and quantum confinement. ${ }^{1,2}$ The value of the effective third-order optical susceptibility of the composite, $\chi^{(3)}$, depends on the metal and the host, and also on several parameters like the size and shape of the clusters and the volume fraction. Values for the intrinsic third-order susceptibility, $\chi_{m}^{(3)}$, of $\mathrm{Cu}$ metallic nanoclusters are in the range $10^{-9}-10^{-7}$ esu. $^{3-7}$ Such high values could be approached for the effective $\chi^{(3)}$ of $\mathrm{Cu}$ composite samples prepared with a high concentration of clusters.

Ion implantation is a very attractive technique for producing such nanocomposite materials with a high concentration of clusters since it can overcome the equilibrium solubility limits. In addition, it is a well-established technique to produce waveguides of very good quality in various materials including silica glasses. ${ }^{8} \mathrm{Cu}$-silica composites produced by ion implantation have already been produced, ${ }^{4}$ showing a quite broad cluster size distribution and $\chi^{(3)}$ values typically in the $10^{-9}$ to $10^{-8}$ esu range. The highest are reached for the largest cluster sizes. ${ }^{6,7,9,10}$ In this article we report on the production of novel $\mathrm{Cu}$ implanted silica samples having a high concentration of clusters of small size and a very narrow size distribution, showing a $\chi^{(3)}$ value of $10^{-7}$ esu and a response time below 2 ps.

Fused silica substrates with a size of $75 \times 25 \mathrm{~mm}$ by 1 $\mathrm{mm}$ thick were implanted to a dose of $8 \times 10^{16} \mathrm{Cu}^{+}$ions/

\footnotetext{
${ }^{a)}$ Electronic mail: j.olivares@io.cfmac.csic.es

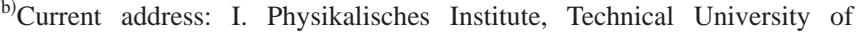
Aachen, Sommerfeldstrasse 14, 52056 Aachen, Germany.
}

$\mathrm{cm}^{2}$. A crucial condition of the implants is that they are thermally bonded to a water cooled sample holder and are maintained at a constant substrate temperature of $20^{\circ} \mathrm{C}$ during the implantation process. The energy used was $50 \mathrm{keV}$, while the ion beam current was $541 \mu \mathrm{A}$ for an ion beam size of 2.5 $\times 5.1 \mathrm{~cm}$ (current density $=42 \mu \mathrm{A} / \mathrm{cm}^{2}$ ). The sample holder was scanned perpendicularly to the ion beam at a speed of 5 $\mathrm{cm} / \mathrm{s}$ in the $x$ direction. There were also incremental steps in the $y$ axis between scans in order to produce a uniform inplane implant profile.

The $\mathrm{Cu}$ profile was calculated using the SUSPRE code which accounts for the significant sputtering taking place. The predicted profile is close to a step function with maximum $\mathrm{Cu}$ concentration at the surface and an estimated full width at half maximum (FWHM) of $40 \mathrm{~nm}$. The implanted samples were characterized with grazing $\mathrm{x}$-ray reflectometry in the angle-dispersive mode, a technique that provides the density and the thickness of thin films. ${ }^{11}$ A thickness value of $(41.0 \pm 0.3) \mathrm{nm}$ was determined in good agreement with the predicted one. The samples were also analyzed by the smallangle $\mathrm{x}$-ray scattering technique under grazing incidence (GISAXS). ${ }^{12,13}$ Figure 1 shows the measured GISAXS pattern for a typical implanted sample. This figure displays a centrosymmetric shape for the scattered intensity distribution, indicating that the clusters are spherical. The intensity can be integrated over a large circular sector, obtaining $I(q)$, where $q$ is the scattering vector. The Guinier plot, ${ }^{14} \log I(q)$ $=\left(f q^{2}\right)$, gives a very well-defined linear part, leading to an average diameter for the clusters of $\phi=(4.0 \pm 0.4) \mathrm{nm}$, and is consistent only with a very narrow size distribution.

Optical transmission and reflection spectra were recorded in the visible spectral region and are shown in Fig. 2. The transmission spectrum shows a weak and broad absorp- 


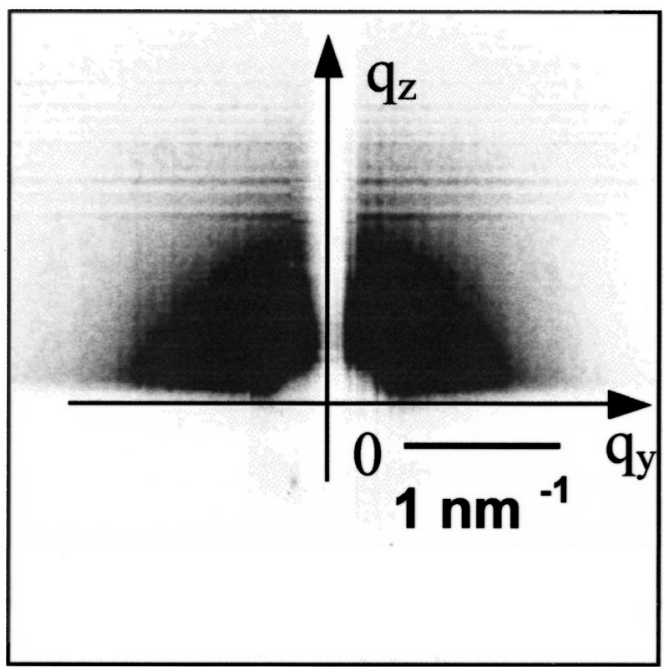

FIG. 1. GISAXS pattern of a $\mathrm{Cu}$ implanted silica sample. The plot shows in a grayscale, the scattered intensity $I(q)$ versus $q=4 \pi \sin \theta / \lambda$, where $\theta$ is the scattering angle and $\lambda$ is the wavelength of the $x$ rays $(1.54 \AA)$.

tion band at $\lambda \approx 580 \mathrm{~nm}$, typical of the surface plasmon resonance (SPR) of $\mathrm{Cu}$ clusters. The dependence of this absorption band on the mean cluster diameter has been reported, and it has been shown that the band becomes noticeable and sharpens only when the diameter is larger than about 5 $\mathrm{nm} .{ }^{7,10}$ The $\mathrm{Cu}$ cluster sizes estimated from the absorption spectra are thus consistent with the values obtained from GISAXS. These measurements also allow one to determine the refraction index $\hat{n}=n+\mathbf{i} k$ of the composite, values of $n$ $=(2.4 \pm 0.1)$ and $k=(0.25 \pm 0.02)$, both parameters showing a large increase with respect to those of the silica matrix.

The nonlinear optical characterization of the samples was carried out by degenerate four-wave mixing (DFWM). The laser source used was a cavity-dumped, synchronously pumped, mode-locked Rhodamine 6G laser, tuned at a wavelength of $585 \mathrm{~nm}$. The laser system provides $13 \mathrm{ps}$ laser pulses in the $10 \mathrm{~nJ} /$ pulse level, at a variable repetition rate. The repetition rate used was $400 \mathrm{kHz}$, low enough to minimize long term thermal effects, and giving a good signal-tonoise ratio with the standard phase lock-in detection technique used. The DFWM experiment was set up in the forward (BOXCARS) scheme ${ }^{15}$ with three parallel incoming beams overlapped at the focus of an achromatic lens of 80

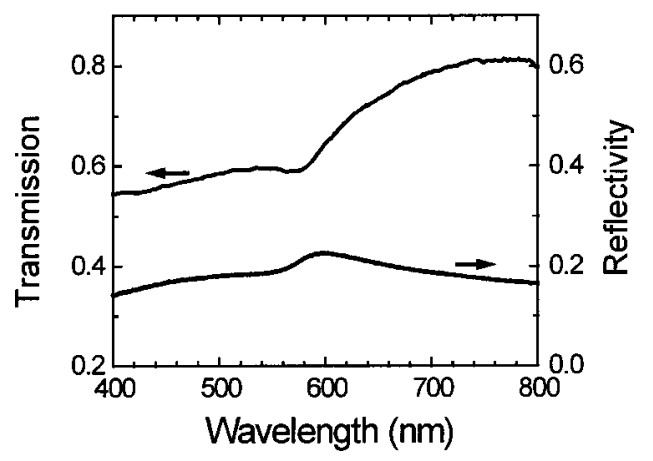

FIG. 2. Transmission and reflection spectra measured for a $\mathrm{Cu}$ implanted silica sample (light beam incident on the implanted side).
TABLE I. Values of the third-order nonlinear optical susceptibility obtained from DFWM measurements under picosecond pulses. The sample effective length is $40 \mathrm{~nm}$ and its refractive index $n=2.4$. For the reference we use a value for the $\mathrm{CS}_{2}$ of $\chi_{x x x x}^{(3)}=2 \times 10^{-12}$ esu.

\begin{tabular}{lccc}
\hline \hline & \multicolumn{2}{c}{$\chi_{i j k l}^{(3)}(-\omega, \omega, \omega,-\omega)$} & \\
& $\chi_{x x x x}^{(3)}(\mathrm{esu})$ & $\chi_{x y x y}^{(3)}(\mathrm{esu})$ & $\chi_{x y y z}^{(3)}(\mathrm{esu})$ \\
\hline Cu: silica & $1.0 \pm 0.5) \times 10^{-7}$ & $(5 \pm 1) \times 10^{-8}$ & $(1.2 \pm 0.5) \times 10^{-8}$ \\
$\mathrm{CS}_{2}$ & $2 \times 10^{-12}$ & $(2.7 \pm 0.3) \times 10^{-13}$ & $(1.4 \pm 0.2) \times 10^{-12}$ \\
\hline \hline
\end{tabular}

$\mathrm{mm}$ focal length. The use of half-wave plates in the beams allowed independent selection of their polarizations so as to measure the different components of the third-order optical nonlinear tensor $\chi_{i j k l}^{(3)}(-\omega ; \omega, \omega,-\omega)$. The conjugated signals were measured for different pump intensities for both the samples and a $\mathrm{CS}_{2}$ cell was used as a reference. ${ }^{16} \mathrm{CS}_{2}$ gave the expected cubic dependence of the conjugated signal across the whole pumping intensity interval studied $\left(10^{7}\right.$ to $10^{8} \mathrm{~W} / \mathrm{cm}^{2}$ ). For the Cu-silica composites the dependence of the conjugated signal on the pump intensity showed some degree of saturation for pump intensities above $3 \times 10^{7} \mathrm{~W} /$ $\mathrm{cm}^{2}$. In order to determine the values of $\left|\chi_{i j k k}^{(3)}\right|$ by comparison with $\mathrm{CS}_{2}$, we selected the data corresponding to the low pump intensity region. The values obtained for the modulus of the different components of $\chi^{(3)}$ under ps pulses are collected in Table I and were obtained using the effective refractive index and absorption coefficient of the composite. Note the remarkably high values obtained for this $\mathrm{Cu}$ composite, where $\chi_{x x x x}^{(3)}=10^{-7} \mathrm{esu}$, which is at least one order of magnitude larger than the earlier reported values for $\mathrm{Cu}$ implants. ${ }^{4,6,10}$

The time evolution of the conjugated signal was measured for the particular case of the response associated to the $\chi_{x y x y}^{(3)}$ component. This corresponds to the use of two parallel polarization, temporally overlapping beams, and one crosspolarized, time-delayed beam. This configuration allowed the best signal-to-noise ratio. Under picosecond pulses, the DFWM signal closely follows the shape of the exciting pulses. In order to explore the response time in the subpicosecond time scale, a single stage fiber-grating pulse compressor was used. ${ }^{17}$ The 13 ps pulses from the dye laser were thus compressed down to $450 \mathrm{fs}$ as determined by means of a standard background-free second harmonic autocorrelation trace. The time evolution of the conjugated signal can be seen in Fig. 3. The signal has a rise time that follows the leading edge of the pulse and decays more slowly than the pulse, giving rise to an asymmetric response curve with a FWHM of 1.4 ps. [Notice that the signal for negative delays and long positive ones $(>2 \mathrm{ps})$ is due to the presence of relatively large uncompressed side wings in the pulse.]

When comparing the present $\mathrm{Cu}$ composites to those reported earlier there are clear differences both in the implantation conditions and the final results. All earlier works report larger cluster sizes, ranging from 5 to $25 \mathrm{~nm}$ depending on the implantation current and energy used. Size distributions are also broader. The energies used were higher, from $60 \mathrm{keV}^{10}$ to $160 \mathrm{keV}^{6,9}$ Under low current conditions the cluster size has been observed to increase with the 


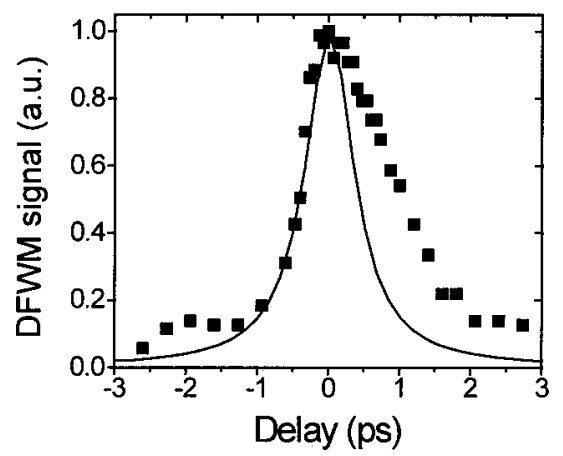

FIG. 3. The time evolution of the DFWM signal measured for a $\mathrm{Cu}$ implanted silica sample with 450 fs pulses is shown with symbols. The line is a Lorentzian fit of the second order autocorrelation trace measured for the pulse.

current. ${ }^{6,79}$ Crucially, the total dose used in those works is lower than here. Regarding the linear optical properties, we obtained an optical density SPR $(\approx 580 \mathrm{~nm})$ of $\mathrm{OD} \approx 0.1$, which is larger than the one obtained in Ref. 6 for clusters of $5 \mathrm{~nm}$. Taking into account the smaller size of the clusters reported here $(4 \mathrm{~nm})$, the absorption value observed indicates that these samples have a larger volume fraction of small clusters $(<5 \mathrm{~nm})$ than that of earlier reports. ${ }^{6,7-10}$ Our specific implantation conditions, a low-energy of $50 \mathrm{keV}$, a high density current of $42 \mu \mathrm{A} / \mathrm{cm}^{2}$, and especially the constant temperature of $20^{\circ} \mathrm{C}$ for the sample, seem responsible for the smaller size of the clusters, their very narrow size distribution, and their higher concentration (i.e., volume fraction). In most of the earlier studies the sample temperature was poorly controlled and there were rapid and significant rises in the surface temperature. Performing the ion implantation at high-current increases the local temperature and additionally the high damage rate provides rapid radiation assisted diffusion. Both factors, which increase the ion mobility, lead to large scale clustering and/or large spread in the size distribution. Such thermal effects would normally be even more evident when the power is deposited in a shallow layer, as in the present case where the ion energy is only $50 \mathrm{keV}$. However, large cluster formation was inhibited, even under the present high-current conditions, by paying particular attention to maintaining the sample at just $20^{\circ} \mathrm{C}$ and by scanning over a large area. Under these conditions one can maintain a high concentration of cluster seeds that form at the very early stages of the implantation process. These nuclei subsequently grow uniformly adsorbing further implanted copper ions.

Since the effective third-order optical susceptibility of the composite, $\chi^{(3)}$, is related to the intrinsic third-order optical susceptibility of the clusters, $\chi_{m}^{(3)}$, via an enhancement field factor $(f)$ and the volume fraction $(p)$, it is thus expected that high values for $\chi^{(3)}$ can be achieved by increasing significantly the volume fraction $p$. Strong enhancement of the intrinsic nonlinear susceptibility due to quantum confinement $\left(\chi_{m}^{(3)} \sim \phi^{-3}\right)$ has also been claimed to occur for small $\mathrm{Cu}$ nanoclusters. ${ }^{5,6}$ The high volume fraction reached combined with the small size of the cluster $(\phi=4 \mathrm{~nm})$ and their very narrow size distributions are most likely responsible for the high performance achieved.

In summary, the use of low-energy high-current $\mathrm{Cu}$ implantation in fused silica at well-controlled low temperature allows one to synthesize novel $\mathrm{Cu}$ nanocomposites having a high density of small clusters with very narrow size distribution, which show a very large $\chi^{(3)}$ value $\left(10^{-7} \mathrm{esu}\right)$ and a response time below $2 \mathrm{ps}$.

This work has been partially supported by the EU under the BRPR-CT98-0616 project, by CAM, Spain, under the 075/0038/1998 project, and by the CSIC-CNRS 2000FR0026 agreement for traveling funding. J.O. wishes to thank CSIC for funding his position. A.L.S. gratefully acknowledges the Alexander von Humboldt foundation and RFBR 99-02-17767 for financial support. The authors thank Dr. D. Babonneau, Dr. R. Serna, and Dr. J. Gonzalo for helpful discussions. The authors also wish to thank the technical staff of LURE Laboratory (Orsay, France) for providing the synchrotron radiation beam and for assistance with the experiments.

${ }^{1}$ D. Ricard, Ph. Roussignol, and C. Flytzanis, Opt. Lett. 10, 511 (1985).

${ }^{2}$ C. Flytzanis, in Beam Shaping and Control with Nonlinear Optics, Vol. 369 of NATO Advanced Science Institute Series, edited by F. Kajzar and R. Reinish (Plenum, New York, 1998), p. 427.

${ }^{3}$ F. Hache, D. Ricard, C. Flytzanis, and U. Kreibig, Appl. Phys. A: Solids Surf. 47, 347 (1988).

${ }^{4}$ R. F. Haglund, Jr., L. Yang, R. H. Magruder III, J. E. Wittig, K. Becker, and R. A. Zuhr, Opt. Lett. 18, 373 (1993).

${ }^{5}$ J. M. Ballesteros, J. Solis, R. Serna, and C. N. Afonso, Appl. Phys. Lett. 74, 2791 (1999)

${ }^{6}$ L. Yang, K. Becker, F. M. Smith, R. H. Magruder III, R. F. Haglund, Jr., L. Yang, R. Dorsinville, R. R. Alfano, and R. A. Zuhr, J. Opt. Soc. Am. B 11, 457 (1994).

${ }^{7}$ R. H. Magruder III, R. F. Haglund, Jr., L. Yang, J. E. Wittig, and R. A. Zuhr, J. Appl. Phys. 76, 708 (1994).

${ }^{8}$ P. D. Townsend, P. J. Chandler, and L. Zhang, Optical Effects of Ion Implantation (Cambridge University, Cambridge, England, 1994), Chap. 6.

${ }^{9}$ M. Falconieri, G. Salvetti, E. Cattaruzza, F. Gonella, G. Mattei, P. Mazzoldi, M. Piovesan, G. Battaglin, and R. Polloni, Appl. Phys. Lett. 73, 288 (1998).

${ }^{10}$ Y. Takeda, V. T. Gritsyna, N. Umeda, C. G. Lee, and N. Kishimoto, Nucl. Instrum. Methods Phys. Res. B 148, 1029 (1999).

${ }^{11}$ A. Naudon, J. Chihab, P. Goudeau, and J. Mimault, J. Appl. Crystallogr. 22, 460 (1989).

${ }^{12}$ A. Naudon and D. Thiaudière, J. Appl. Crystallogr. 30, 822 (1997).

${ }^{13}$ A. Naudon, D. Babonneau, D. Thiaudière, and S. Lequien, Physica B 283, 69 (2000).

${ }^{14}$ A. Guinier and G. Fournet, Small-Angle Scattering of X-rays (Chapman and Hall, London, 1955).

${ }^{15}$ A. L. Smirl, T. F. Bogges, and A. Hopf, Opt. Commun. 34, 463 (1980).

${ }^{16}$ R. L. Sutherland, Handbook of Nonlinear Optics (Marcel Dekker, New York, 1996), Chap. 7.

${ }^{17}$ B. Nikolaus and D. Grischkowsky, Appl. Phys. Lett. 43, 228 (1983).

${ }^{18}$ D. E. Hole, P. D. Townsend, J. D. Barton, L. C. Nistor, and J. van Landuyt, J. Non-Cryst. Solids 180, 266 (1995). 